\title{
MAPT rs242562 and GSK3B rs334558 are associated with Parkinson's Disease in central China
}

\author{
Lan $\mathrm{Yu}^{1+}$, Jinsha Huang ${ }^{1 \dagger}$, Desheng Zhai ${ }^{4+}$, Ling Liu', Kexin Guo ${ }^{1}, \mathrm{Xi} \mathrm{Long}^{2}$, Jing Xiong ${ }^{3}$, Zhentao Zhang ${ }^{3}$, \\ Youpei Wang ${ }^{1}$, Ying Zhao ${ }^{5}$, Ping $\mathrm{Wu}^{6}$, Dingan Wang ${ }^{6}$, Zhicheng $\mathrm{Lin}^{7,8,9}$, Jing $\mathrm{Wu}^{10}$, Nian Xiong ${ }^{{ }^{*}}$ and Tao Wang ${ }^{1 *}$
}

\begin{abstract}
Background: Microtubule-associated protein tau (MAPT) is a neuronal protein involved in the pathogenesis of several neurodegenerative diseases including Parkinson's Disease (PD). Glycogen synthase kinase 3 beta (GSK3B) catalyzes phosphorylation in multiple sites of tau protein. However, whether or not there is any association between the GSK3B gene alteration, MAPT haplotype and PD remains unexplored in Chinese population, especially in central Chinese population.

Results: Here, we aimed at studying the effect of MAPT rs 242562 and GSK3B rs334558 on the risk of PD by performing a case-control association study in central China. Our data showed that all PD patients and controls were of MAPT H1/ $\mathrm{H} 1$ diplotype in our study, thus confirming that the distribution of the MAPT H1 haplotype is common in China. GG genotype of MAPT rs242562 serves protection effect on PD risk in central Chinese population, while genotype of GSK3B rs334558 showed no difference between PD patients and controls.
\end{abstract}

Conclusions: We conclude that the MAPT rs242562 is associated with PD in central China in the background of MAPT H1/H1 diplotype. The GG genotype of rs 242562 displays protection against PD in subgroup with GSK3B rs334558 T carrier.

Keywords: MAPT H1 haplotype, GSK3 $\beta$, Parkinson's disease, Genetic risk factor

\section{Background}

Parkinson's disease (PD) is a common neurodegenerative disease in elders, characterized by the loss of dopaminergic neurons in substantial nigra and the formation of Lewy bodies [1]. PD affects approximately $1 \%$ of population aging over 60 years. Disease onset before 50 years of age defines as early-onset Parkinson's disease (EOPD) while that over 50 years of age, as late onset Parkinson's disease (LOPD). The etiology and pathogenesis of PD, mainly considered to be related with aging, genetic and environmental factors, is incompletely understood. Recently, linkage analysis and genomewide association studies have shown that PARK1-PARK18, POLG, Glycogen

\footnotetext{
* Correspondence: nianxiongtjmu@gmail.com; wangtaowh@hust.edu.cn ${ }^{\dagger}$ Equal contributors

'Department of Neurology, Union Hospital, Tongji Medical College, Huazhong University of Science and Technology, 1277 Jiefang Road, Wuhan 430022, Hubei, China

Full list of author information is available at the end of the article
}

Synthase kinase-3B (GSK3B), Microtubule-associated protein tau (MAPT) and other genes are associated with PD risk.

The MAPT gene is localized in a region of extended linkage disequilibrium (LD) on chromosome 17q21, containing 16 exons and encoding microtubule-associated proteins tau. Tau proteins, constituting a family of six isoforms ranging between $352-441$ amino acids, are widely expressed in the nervous system. Excessive phosphorylation of tau proteins leads to abnormal intracellular aggregation, formatting of the double helix fiber filament, producing the nerve fiber tangles, and finally neurodegeneration [2]. MAPT gene, defines two extended haplotype, $\mathrm{H} 1$ and $\mathrm{H} 2$. The determination of MAPT haplotype $\mathrm{H} 1 / \mathrm{H} 2$ is attributed to the presence of a 238 bp deletion between exons 9 and 10 of MAPT H2 haplotype [3]. H1 haplotype is reportedly related to PD susceptibility [4]. Concerning sub-haplotypes, there is a positive correlation between MAPT H1 haplotype and PD, including SNP rs242562 (A/G) and rs2435207 (G/A). The A-A sub-haplotype is 
associated with PD patients in a Norwegian population, while the G-A sub-haplotype for these two SNPs is associated with PD subjects in Greece [5-7]. However, at present, little is known about the correlation between $M A P T$ gene polymorphism and PD patients in China, especially in central China area. Central China includes six widely agricultural provinces namely Hunan, Hubei, Jiangxi, Henan, Shanxi and Anhui.

GSK3B protein is widely expressed in all tissues, with particularly abundant levels in the brain [8]. The fundamental role of GSK3B in intracellular neuronal signaling systems is underpinned by its ability to phosphorylate several proteins that contribute to the structural characteristics and dynamics of neuronal cells. In addition, it has been proven that GSK3B catalyzes phosphorylation in multiple sites of tau protein [9], and it is an important pathogenic protein kinase for PD. Previous data indicate that the genetic alteration of GSK3B and its interaction with MAPT haplotypes are collectively related to PD morbidity rate in a Greek cohort $[3,10]$. However, the association between the GSK3B gene alteration, MAPT haplotype and PD has not been previously explored in Chinese population.

In this study, our database contains 211 PD patients and 279 controls from central China. Our study examined the possible association between GSK3B promoter single nucleotide polymorphism (SNP) rs334558, MAPT haplotype, $M A P T$ intron SNP rs242562 and PD in central China.

\section{Methods}

\section{Subjects}

We recruited 211 unrelated sporadic PD patients (average age at diagnosis: $57.23 \pm 12.9$ years; 137 males, 74 females) diagnosed according to the criteria of the UK Parkinson's Disease Society Brain Bank in Union hospital, Tongji Medical College, Huazhong University of Science and Technology (TJMC \& HUST). The control group consisted of 279 individuals (average age: $53.72 \pm 13.32$ years; 178 males, 101 females). This study was approved by the Ethical Committee of TJMC \& HUST. The ethics and written informed consent was obtained from all subjects.

\section{Genotyping}

Genomic DNA was extracted from venous blood using standard methods. Our primary objective was to select $M A P T$ polymorphisms previously suggested to contribute to the risk of developing idiopathic PD in Chinese subjects. Therefore, we focused on the H1/H2 insertion/ deletion polymorphism and rs242562 [11]. PCR products were generated with $50 \mathrm{ng}$ DNA template in $12.5 \mu \mathrm{l} 1 \times$ taq PCR Master MIX (Bioteke Corporation, Beijing, China), $1 \mu \mathrm{l}$ of $10 \mu \mathrm{mol} / \mathrm{l}$ each primer (Invitrogen, Carlsbad, CA, USA) and $12 \mu \mathrm{l}$ Ultra-distilled water in a total volume of $25 \mu$ l. The PCR conditions used for the H1/H2 insertion/ deletion polymorphism were: the $\mathrm{H} 1 / \mathrm{H} 2$ insertion/deletion polymorphism forward primer GGAAGACGTTCTCACT GATCTG, reverse primer AGGAGTCTGGCTTCAGTC TCTC, initial denaturation at $94^{\circ} \mathrm{C}$ for 5 minutes followed by 33 cycles of $94^{\circ} \mathrm{C}$ for 30 seconds, $59^{\circ} \mathrm{C}$ for 30 seconds, $72^{\circ} \mathrm{C}$ for 30 seconds, and a final extension at $72^{\circ} \mathrm{C}$ for 10 minutes. The PCR conditions used for the rs 242562 polymorphism were: rs242562 forward primer: CAGCCT TCCCTGTCCTTGATTC, rs242562 reverse primer; GCC TTCCCAACAGAGCAACC, initial denaturation at $94^{\circ} \mathrm{C}$ for 5 minutes, followed by 35 cycles of $94^{\circ} \mathrm{C}$ for 30 seconds, $58^{\circ} \mathrm{C}$ for 30 seconds and $72^{\circ} \mathrm{C}$ for 30 seconds, and a final extension at $72^{\circ} \mathrm{C}$ for 10 minutes. Digestion with XhoI restriction enzyme (New England Biolabs, Ipswich, MA, USA) at $37^{\circ} \mathrm{C}$ yielded a $385 \mathrm{bp}$ band for the G-allele and a $287 \mathrm{bp}$ and a $98 \mathrm{bp}$ for the A-allele. The PCR conditions used for the rs334558 polymorphism were: rs334558 forward primer: GACGTCCGTGATT GGCTC, reverse primer: AGCCCAGAGCCCTGTCAG, initial denaturation at $94^{\circ} \mathrm{C}$ for 5 minutes, followed by 33 cycles of $94^{\circ} \mathrm{C}$ for 30 seconds, $62^{\circ} \mathrm{C}$ for $30 \mathrm{~s}$ and $72^{\circ} \mathrm{C}$ for 15 seconds, with a final extension at $72^{\circ} \mathrm{C}$ for 10 minutes [12]. Digestion with AluI restriction enzyme (New England Biolabs) at $37^{\circ} \mathrm{C}$ yields a 344 bp band for the $\mathrm{C}$ allele and 220 and $124 \mathrm{bp}$ for the T allele. Digestion products were resolved on a $2 \%$ agarose gel, stained in ethidium bromide solution and visualized with an ultraviolet light.

\section{Statistical analysis}

All statistical analysis was performed by SAS 9.2 (SAS Institute, Cary, NC). A log-odds ratio (OR) and its $95 \%$ confidence interval (CI) were estimated with logistic regression for allele wise, genotype-wise recessive/ dominant genetic model of each polymorphism. The gene-gene interaction test was conducted by running two models: one with and the other without the interaction term (A-carrier * T-carrier). In addition, a trend test in logistic regression for allelic model was further employed for the effect of MAPT rs242562 and GSK3B rs334558 on risk for PD [13]. A probability $(P)$ of less than 0.05 is considered statistically significant and $P$-values are two-tailed. Adjustment of $P$ values for multiple testing uses Bonferroni correction. The power was calculated by Power V3.0 software (http://dceg.cancer.gov/tools/design/ POWER) with the observed odds rates and minor allele frequencies for both the 2-df overall association test and the trend test [14].

\section{Results}

The allele and genotype distribution of MAPT rs242562 and GSK3B rs334558 in PD patients and controls were summarized in Table 1. The schematic of the MAPT region and associated polymorphisms including rs242562 
Table 1 Allele and Genotype frequencies of MAPT rs242562 and GSK3B rs334558 in PD cases and controls

\begin{tabular}{|c|c|c|c|c|c|c|c|c|c|c|c|c|}
\hline & \multicolumn{6}{|c|}{ Case(211) } & \multicolumn{6}{|c|}{ Control(279) } \\
\hline & Number & $\%$ & Number & $\%$ & Number & $\%$ & Number & $\%$ & Number & $\%$ & Number & $\%$ \\
\hline \multicolumn{13}{|l|}{ rs242562 } \\
\hline Allele & A & & G & & & & A & & G & & & \\
\hline Total & 230 & 55.02 & 188 & 44.98 & & & 225 & 48.91 & 235 & 51.09 & & \\
\hline Male & 149 & 54.78 & 123 & 45.22 & & & 140 & 48.61 & 148 & 51.39 & & \\
\hline Female & 81 & 55.48 & 65 & 44.52 & & & 85 & 49.42 & 87 & 50.58 & & \\
\hline Age $<=50$ & 80 & 54.05 & 68 & 45.95 & & & 102 & 43.97 & 130 & 56.03 & & \\
\hline Age $>50$ & 150 & 55.56 & 120 & 44.44 & & & 123 & 53.95 & 105 & 46.05 & & \\
\hline Genotype & AA & & $A G$ & & GG & & AA & & $A G$ & & GG & \\
\hline Total & 57 & 27.27 & 116 & 55.5 & 36 & 17.22 & 59 & 25.65 & 107 & 46.52 & 64 & 27.83 \\
\hline Male & 37 & 27.21 & 75 & 55.15 & 24 & 17.65 & 36 & 25 & 68 & 47.22 & 40 & 27.78 \\
\hline Female & 20 & 27.4 & 41 & 56.16 & 12 & 16.44 & 23 & 26.74 & 39 & 45.35 & 24 & 27.91 \\
\hline Age $<=50$ & 20 & 27.23 & 40 & 54.05 & 14 & 18.92 & 27 & 23.28 & 48 & 41.38 & 41 & 35.34 \\
\hline Age $>50$ & 37 & 27.41 & 76 & 56.3 & 22 & 16.3 & 32 & 28.07 & 59 & 51.75 & 23 & 20.18 \\
\hline \multicolumn{13}{|l|}{ rs334558 } \\
\hline Allele & $C$ & & T & & & & C & & T & & & \\
\hline Total & 260 & 61.61 & 162 & 38.39 & & & 330 & 62.98 & 194 & 37.02 & & \\
\hline Male & 170 & 62.04 & 104 & 37.96 & & & 218 & 63.74 & 124 & 36.26 & & \\
\hline Female & 90 & 60.81 & 58 & 39.19 & & & 112 & 61.54 & 70 & 38.46 & & \\
\hline Age $<=50$ & 97 & 65.54 & 51 & 34.46 & & & 163 & 65.73 & 85 & 34.27 & & \\
\hline Age $>50$ & 163 & 59.49 & 111 & 40.51 & & & 167 & 60.51 & 109 & 39.49 & & \\
\hline Genotype & $C C$ & & $\mathrm{CT}$ & & $\pi$ & & $C C$ & & $C T$ & & $\pi$ & \\
\hline Total & 74 & 35.07 & 112 & 53.08 & 25 & 11.85 & 97 & 37.02 & 136 & 51.91 & 29 & 11.07 \\
\hline Male & 53 & 38.69 & 64 & 46.72 & 20 & 14.6 & 62 & 36.26 & 94 & 54.97 & 15 & 8.77 \\
\hline Female & 21 & 28.38 & 48 & 64.86 & 5 & 6.76 & 35 & 38.46 & 42 & 46.15 & 14 & 15.38 \\
\hline Age $<=50$ & 27 & 36.49 & 43 & 58.11 & 4 & 5.41 & 52 & 41.94 & 59 & 47.58 & 13 & 10.48 \\
\hline Age $>50$ & 47 & 34.31 & 69 & 50.36 & 21 & 15.33 & 45 & 32.61 & 77 & 55.8 & 16 & 11.59 \\
\hline
\end{tabular}

and rs2435207 were shown in Figure 1. Genotype distributions of rs242562 and rs334558 followed Hardy-Weinberg equilibrium for PD patients and controls, no deviations from equilibrium were observed. All the PD patients and controls were of $\mathrm{H} 1 / \mathrm{H} 1$ diplotype in our study.

For $M A P T$ rs242562, we found positive results for genotype-wise dominant genetic model in involved subjects (Table 2, shown in bold) with a power of $74.5 \%$.
Genotype AG + AA is associated with the higher PD risk in overall involved subjects (AG + AA vs. GG, OR = 1.785, $P=0.016$ ), compared to genotype $\mathrm{GG}$, with $\mathrm{A}$ as the "putative" risk allele (Table 2). These data suggest that AG + AA genotype of rs242562 may confer risk effect on PD risk in central Chinese population. The ORs and 95\% CIs for the case-control studies were not significant for allele-wise model and the recessive model in overall

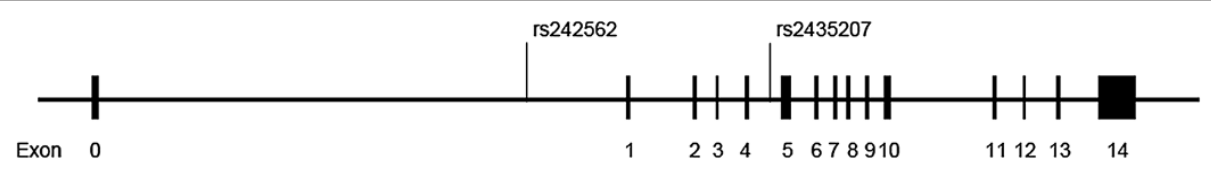

Figure 1 Schematic of the MAPT region and associated polymorphisms. Previous studies have identified a $\sim 970 \mathrm{~kb}$ inversion polymorphism at chromosome 17q21.31, a region that contains MAPT and several other genes. Chromosomes with the inverted segment in different orientations represent two highly divergent MAPT haplotypes, $\mathrm{H} 1$ (direct orientation) and $\mathrm{H} 2$ (inverted orientation). No recombination has been identified between these two haplotypes over a region of $\sim 1.5 \mathrm{Mb}$, but they have accumulated sequence variation independently. Polymorphisms including rs 242562 and rs2435207 spanning MAPT exons 1 to 4 are specific to the $\mathrm{H} 1$ haplotype. Besides, there is a characteristic 238-bp deletion in the MAPT intron 9 of the $\mathrm{H} 2$ haplotype. 
Table 2 Effect of MAPT rs242562 and GSK3B rs334558 on risk for PD

\begin{tabular}{|c|c|c|c|c|c|c|c|c|c|c|}
\hline SNPs & OR $(95 \% \mathrm{Cl})$ & $P$ & Crude OR $(95 \% \mathrm{Cl})$ & $P$ & Adjusted OR $(95 \% \mathrm{Cl})^{*}$ & $P$ & Crude OR $(95 \% \mathrm{Cl})$ & $P$ & Adjusted OR $(95 \% \mathrm{Cl})^{*}$ & $P$ \\
\hline \multirow[t]{2}{*}{ rs242562 (A/G) } & Allele-wise A vs. G & & $A A$ vs. $A G+G G$ & & $A A$ vs. $A G+G G$ & & $A G+A A$ vs. GG & & $A G+A A$ vs. GG & \\
\hline & $1.278(0.980-1.666)$ & 0.071 & $1.087(0.711-1.662)$ & 0.7 & $1.065(0.693-1.636)$ & 0.775 & $1.853(1.169-2.936)$ & 0.009 & $1.785(1.116-2.853)$ & 0.016 \\
\hline Power & 0.266 & & 0.058 & & 0.048 & & 0.793 & & 0.745 & \\
\hline \multirow[t]{2}{*}{ rs334558 (T/C) } & Allele-wise T vs. C & & TT vs. CT + CC & & TT vs. $C T+C C$ & & $C T+\pi$ vs.CC & & $C T+T T$ vs. $C C$ & \\
\hline & $1.060(0.814-1.381)$ & 0.667 & $1.080(0.612-1.907)$ & 0.79 & $1.018(0.573-1.808)$ & 0.951 & $1.088(0.746-1.588)$ & 0.661 & $1.058(0.719-1.557)$ & 0.774 \\
\hline Power & 0.05 & & 0.044 & & 0.029 & & 0.064 & & 0.064 & \\
\hline
\end{tabular}

${ }^{*}$ All the $\mathrm{OR}$ and $P$ values were adjusted for age and gender. 
involved subjects with a lower power of 0.048-0.266 (Table 2). Additionally, the test for trend in logistic regression for allelic model also found no significant association with a power of 0.143 (Table 3), which is consistent with the results for allelic model in Table 2.

For GSK3B rs334558, the allele-wise and genotype-wise ORs lack any statistical significance in overall involved subjects with an insufficient power of 0.029-0.064 (Table 2). The test for trend in logistic regression for allelic model with a power of 0.036 (Table 3) also found null association consistent with the allele-wise model in Table 2.

The gene-gene interaction test was conducted by running two models: with and without the interaction term (A-carrier ${ }^{*} \mathrm{~T}$-carrier), and likelihood ratio test indicated that interaction term was not statistically significant $(P=0.2140)$. The effect of GG and age on PD risk remained the same between the two models. We then studied the association between rs242562 and PD at different levels of the rs334558 genotype (Additional file 1: Table S1), and the association between rs 334558 and PD at different levels of the rs242562 genotype (Additional file 2: Table S2). The results suggest that the GG genotype of rs242562 displays protection against PD in subgroup with $T$ carrier, although the stratified analysis has a smaller sample size and a lower statistic power.

\section{Discussion}

In this study, we investigated the association of MAPT rs242562 and GSK3B rs334558 with PD in central China. The main findings of our study in central Chinese population include 1) All the PD patients and controls were in H1/H1 diplotype in our study, 2) GG genotype of MAPT rs242562 serves protection effect on PD risk in central Chinese population, 3) Genotype of GSK3B rs334558 showed no difference between PD patients and controls, and 4) GG genotype of rs242562 may display strong protective effect against PD risk in subgroup with $G S K 3 B$ rs334558 T carrier.
Our data confirmed that the distribution of the MAPT H1 haplotype is common in China, which is in agreement with previous findings $[15,16]$. Accumulating evidence showed that MAPT $\mathrm{H} 1$ haplotype-carrier group is a susceptible group of PD [16,17]. This case-control study served as an association study based on population with susceptible haplotype $\mathrm{H} 1$ in central China. MAPT rs242562 regulates protein encoding despite it is in the intron of MAPT gene. The frequency of rs 242562 GG genotype is higher in controls compared to that in cases, indicating it may play a protective role. These data are different from a previous study which showed that $M A P T$ polymorphism rs242562 revealed no significant difference between PD patients and controls in Germany, Serbia and Greece $[6,18,19]$. The sample size, ethnic groups and environment may contribute to the different results.

GSK3B rs334558 is reportedly associated with PD, but the conclusions were inconsistent. A Greek study was the first to show that $G S K 3 B$ rs334558 was related to $\mathrm{PD}$, while CC served a protective effect and TT was overexpressed in PD [18]. Moreover, a study from Australia showed that the frequency of TT and H1/H1 diplotype in PD patients was significantly higher compared to control subjects [10]. However, the study about LOPD patients in India concluded that CC was a risk factor [20]. In the Spanish population, TT genotype and MAPT H1/H1 diplotype were associated with a decreased risk for PD [21]. In the present study, we did not find any significant difference in allele-wise and genotype-wise analysis for rs 334558 between PD patients and controls. However, GG genotype of rs242562 may serve a strong protective effect on PD risk in subgroup with $\mathrm{T}$ carrier, although the stratified analysis has a small sample size and a low statistic power.

Sex may be involved in the association results of GSK3B and neurodegenerative disease [22]. In an Alzheimer's disease research, it was revealed that estrogen receptor and GSK3B could change the tau protein phosphorylation,

Table 3 The allelic-wise analysis with a test for trend for the effect of MAPT rs242562 and GSK3B rs334558 on risk for PD

\begin{tabular}{|c|c|c|c|c|c|c|}
\hline Number of minor alleles & Cases & Controls & Crude OR $(95 \% \mathrm{Cl})$ & $P$ value & Adjusted OR $(95 \% \mathrm{Cl})^{*}$ & $\mathrm{P*}$ value \\
\hline \multicolumn{7}{|l|}{ rs242562 } \\
\hline 0 & 36 & 64 & 1.000 & & 1.000 & \\
\hline 1 & 116 & 107 & $1.927(1.186,3.132)$ & 0.008 & $1.892(1.154,3.102)$ & 0.011 \\
\hline 2 & 56 & 60 & $1.659(0.960,2.868)$ & 0.070 & $1.628(0.931,2.846)$ & 0.087 \\
\hline Test for trend & & & $1.262(0.964,1.651)$ & 0.091 & $1.250(0.950,1.646)$ & 0.111 \\
\hline \multicolumn{7}{|l|}{ rs334558 } \\
\hline 0 & 74 & 97 & 1.000 & & 1.000 & \\
\hline 1 & 112 & 136 & $1.079(0.729,1.598)$ & 0.702 & $1.051(0.704,1.570)$ & 0.807 \\
\hline 2 & 25 & 29 & $1.130(0.611,2.089)$ & 0.697 & $1.091(0.583,2.039)$ & 0.785 \\
\hline Test for trend & & & $1.068(0.806,1.415)$ & 0.647 & $1.047(0.785,1.395)$ & 0.756 \\
\hline
\end{tabular}


indicating sex involved in pathogenesis of this neurodegenerative disease [22]. However, following sex stratification the sample size was smaller in our study, and whether hormonal or hormonal receptors change the association of GSK3B gene with PD needs to be further investigated.

Although MAPT rs242562 and GSK3B rs334558 are located in two different chromosomes, the encoded proteins interact with each other. Given the findings that H1 haplotype may act synergistically with variants in the $M A P T$ and GSK3B genes in conferring risk for PD, gene-gene interactions will also be important to consider as they may provide critical insights into mechanisms of disease susceptibility. In this study, lower power was observed for rs 334558 analysis. Thus, the null association should attribute to the insufficient power. Since the sample size of the genotype model including indicator variables of the two polymorphisms is considerably smaller, a larger sample size should be needed in order to further assess this association.

\section{Conclusions}

In summary, the MAPT rs 242562 is associated with PD in central China, and GG genotype of rs242562 may provide protective effect against PD risk in subgroup with GSK3B rs334558 T carrier, although our results are limited by the sample size. Since MAPT gene and GSK3B gene confer genetic risk for PD, exploring how the gene-gene or gene-environment interactions contribute to dopaminergic neurodegeneration should be further considered. Such knowledge about the mechanisms could open up new windows for early diagnostic and therapeutic interventions in this important neurodegenerative disease.

\section{Consent}

Written informed consent was obtained from all patient and control for the association study. A copy of the written consent is available for review by the Series Editor of this journal.

\section{Additional file}

Additional file 1: Table S1. MAPT rs 242562 A/G and risk of PD, by GSK3B rs334558 C/T.

Additional file 2: Table S2. GSK3B rs334558 C/T and risk of PD, by MAPT rs $242562 \mathrm{~A} / \mathrm{G}$.

\section{Abbreviations}

MAPT: Microtubule-associated protein tau; PD: Parkinson's disease LOPD: Late onset of Parkinson's disease; EOPD: Early onset of Parkinson's disease; GSK3B: Glycogen synthase kinase 3ß; TJMC \& HUST: Tongji medical college, Huazhong University of Science; OR: Odds ratios.

\section{Competing interests}

We confirm that we have read the Journal's position on issues involved in ethical publication and affirm that this study is consistent with those guidelines. None of the authors have any conflict of interest to disclose.

\section{Authors' contributions}

$L Y, J H, D Z, K G, N X, X L, J X, Y Z, P W, Z L, J W$, TW contributed to the conception and design. $L Y, D Z, K G, J X, Y W, P W, J H, Z Z$ took care of the PCR studies. LY, $J H, D Z, K G, L L, X L, J X, Y Z, Z L, D W, J W, N X, T W$ analyzed and interoperated the data. $L Y, D Z, X L, L L, Y Z, Z L, D W, J W, N X$, TW coordinated all the experiments and helped to draft the manuscript. All authors read, revised and approved the final manuscript.

\section{Acknowledgments}

This work was supported by grants 30870866, 81071021 and 31171211 from the National Natural Science Foundation of China (to TW), grant 81200983 from the National Natural Science Foundation of China (to NX), grant 81100958 from the National Natural Science Foundation of China (to ZTZ), grant 81301082 from the National Natural Science Foundation of China (to JSH), grant 2012B09 from China Medical Foundation (to NX) and grant 0203201343 from Hubei Molecular Imaging Key Laboratory (to NX). The funders had no role in study design, data collection and analysis, decision to publish, or preparation of the manuscript.

\section{Author details}

'Department of Neurology, Union Hospital, Tongji Medical College, Huazhong University of Science and Technology, 1277 Jiefang Road, Wuhan 430022, Hubei, China. ${ }^{2}$ Department of Radiology, Union Hospital, Tongji Medical College, Huazhong University of Science and Technology, Hubei 430022, China. ${ }^{3}$ Department of Neurology, Renmin Hospital of Wuhan University, Wuhan 430060, China. ${ }^{4}$ Department of Public Health, Xinxiang Medical University, Xinxiang 453003, China. ${ }^{5}$ School of Pharmacy, Xinxiang Medical University, Henan 453003, China. ${ }^{6}$ Hefeng Central Hospital, Hefeng, Enshi, Hubei 445800, China. ${ }^{7}$ Department of Psychiatry, Harvard Medical School, Boston, MA 02114, USA. ${ }^{8}$ Laboratory of Psychiatric Neurogenomics, Division of Alcohol and Drug Abuse, and Mailman Neuroscience Research Center, McLean Hospital, Belmont, MA 02478, USA. ${ }^{9}$ Harvard NeuroDiscovery Center, Boston, MA 02114, USA. ${ }^{10}$ Key Laboratory of Environment and Health, Ministry of Education \& Department of Epidemiology and Biostatistics, School of Public Health, Tongji Medical College, Huazhong University of Science and Technology, Hubei 430030, China.

Received: 9 April 2014 Accepted: 23 April 2014

Published: 29 April 2014

\section{References}

1. Forno LS: Neuropathology of Parkinson's disease. J Neuropathol Exp Neurol 1996, 55(3):259-272.

2. Ballatore C, Lee VM, Trojanowski JQ: Tau-mediated neurodegeneration in Alzheimer's disease and related disorders. Nat Rev Neurosci 2007 8(9):663-672.

3. Kalinderi K, Fidani L, Katsarou Z, Clarimon J, Bostantjopoulou S, Kotsis A: GSK3beta polymorphisms, MAPT H1 haplotype and Parkinson's disease in a Greek cohort. Neurobiol Aging 2011, 32(3):546. e541-545.

4. Baker M, Litvan I, Houlden H, Adamson J, Dickson D, Perez-Tur J, Hardy J, Lynch T, Bigio E, Hutton M: Association of an extended haplotype in the tau gene with progressive supranuclear palsy. Hum Mol Genet 1999, 8(4):711-715.

5. Fidani L, Kalinderi K, Bostantjopoulou S, Clarimon J, Goulas A, Katsarou Z, Hardy J, Kotsis A: Association of the Tau haplotype with Parkinson's disease in the Greek population. Mov Disord 2006, 21(7):1036-1039.

6. Refenes N, Bolbrinker J, Tagaris G, Orlacchio A, Drakoulis N, Kreutz R: Role of the $\mathrm{H} 1$ haplotype of microtubule-associated protein tau (MAPT) gene in Greek patients with Parkinson's disease. BMC Neurol 2009, 9:26.

7. Skipper L, Wilkes K, Toft M, Baker M, Lincoln S, Hulihan M, Ross OA, Hutton M, Aasly J, Farrer M: Linkage disequilibrium and association of MAPT H1 in Parkinson disease. Am J Hum Genet 2004, 75(4):669-677.

8. Embi N, Rylatt DB, Cohen P: Glycogen synthase kinase-3 from rabbit skeletal muscle, Separation from cyclic-AMP-dependent protein kinase and phosphorylase kinase. Eur J Biochem 1980, 107(2):519-527. 
9. Rankin CA, Sun Q, Gamblin TC: Tau phosphorylation by GSK-3beta promotes tangle-like filament morphology. Mol Neurodegener 2007, 2:12.

10. Kwok JB, Hallupp M, Loy CT, Chan DK, Woo J, Mellick GD, Buchanan DD, Silburn PA, Halliday GM, Schofield PR: GSK3B polymorphisms alter transcription and splicing in Parkinson's disease. Ann Neurol 2005, 58(6):829-839.

11. Wider C, Vilarino-Guell C, Heckman MG, Jasinska-Myga B, Ortolaza-Soto Al, Diehl NN, Crook JE, Cobb SA, Bacon JA, Aasly JO, Gibson JM, Lynch T, Uitti RJ, Wszolek ZK, Farrer MJ, Ross OA: SNCA, MAPT, and GSK3B in Parkinson disease: a gene-gene interaction study. Eur J Neurol 2011, 18(6):876-881.

12. Yuan $Y$, Tong $Q$, Zhou $X$, Zhang $R$, Qi Z, Zhang K: The association between glycogen synthase kinase 3 beta polymorphisms and Parkinson's disease susceptibility: a meta-analysis. Gene 2013, 524(2):133-138.

13. Guedj M, Nuel G, Prum B: A note on allelic tests in case-control association studies. Ann Hum Genet 2008, 72(Pt 3):407-409.

14. Faul F, Erdfelder E, Lang AG, Buchner A: G Power 3: a flexible statistical power analysis program for the social, behavioral, and biomedical sciences. Behav Res Methods 2007, 39(2):175-191.

15. Evans W, Fung HC, Steele J, Eerola J, Tienari P, Pittman A, Silva R, Myers A, Vrieze FW, Singleton A, Hardy J: The tau $\mathrm{H} 2$ haplotype is almost exclusively Caucasian in origin. Neurosci Lett 2004, 369(3):183-185.

16. Zhang J, Song Y, Chen H, Fan D: The tau gene haplotype h1 confers a susceptibility to Parkinson's disease. Eur Neurol 2005, 53(1):15-21.

17. Healy DG, Abou-Sleiman PM, Lees AJ, Casas JP, Quinn N, Bhatia K, Hingorani AD, Wood NW: Tau gene and Parkinson's disease: a case-control study and meta-analysis. J Neurol Neurosurg Psychiatry 2004, 75(7):962-965.

18. Landrigan PJ, Sonawane B, Butler RN, Trasande L, Callan R, Droller D: Early environmental origins of neurodegenerative disease in later life. Environ Health Perspect 2005, 113(9):1230-1233.

19. Winkler S, Konig IR, Lohmann-Hedrich K, Vieregge P, Kostic V, Klein C: Role of ethnicity on the association of MAPT H1 haplotypes and subhaplotypes in Parkinson's disease. Eur J Hum Genet 2007, 15(11):1163-1168.

20. Das G, Misra AK, Das SK, Ray K, Ray J: Role of tau kinases (CDK5R1 and GSK3B) in Parkinson's disease: A study from India. Neurobiol Aging 2012, 33(7):1485. e9-15.

21. Garcia-Gorostiaga I, Sanchez-Juan P, Mateo I, Rodriguez-Rodriguez E, Sanchez-Quintana C, del Olmo SC, Vazquez-Hiquera JL, Berciano J, Combarros O, Infante J: Glycogen synthase kinase-3 beta and tau genes interact in Parkinson's and Alzheimer's diseases. Ann Neurol 2009, 65(6):759-761. author reply 761-752

22. Goodenough S, Schleusner D, Pietrzik C, Skutella T, Behl C: Glycogen synthase kinase 3 beta links neuroprotection by 17 beta-estradiol to key Alzheimer processes. Neuroscience 2005, 132(3):581-589.

\section{Submit your next manuscript to BioMed Central and take full advantage of:}

- Convenient online submission

- Thorough peer review

- No space constraints or color figure charges

- Immediate publication on acceptance

- Inclusion in PubMed, CAS, Scopus and Google Scholar

- Research which is freely available for redistribution 